\title{
PUBLICATIONS
}

Bulletin of the American Mathematical Society.

Transactions of the American Mathematical Society.

Mathematical Papers of the Chicago Congress, 1893.

Evanston Colloquium Lectures, 1893, by Felix Klein.

\section{Colloquium Series}

Volume 1. The Boston Colloquium, by H. S. White, F. S. Woods, and E. B. Van Vleck. 1905.

Volume 2. The New Haven Colloquium. Out of print.

Volume 3. The Princeton Colloquium, by G. A. Bliss and E. Kasner. 1913. Reprinted, 1934.

Volume 4. The Madison Colloquium, by L. E. Dickson and W. F. Osgood. 1914.

Volume 5. The Cambridge Colloquium. Part I. Out of print. Part II: Analysis Situs, by O. Veblen. Second edition, 1931.

Volume 6. The Logarithmic Potential. Discontinuous Dirichlet and Neumann Problems, by G. C. Evans. 1927.

Volume 7. Algebraic Arithmetic, by E. T. Bell. 1927.

Volume 8. Non-Riemannian Geometry, by L. P. Eisenhart. 1927.

Volume 9. Dynamical Systems, by G. D. Birkhoff. 1927.

Volume 10. Algebraic Geometry and Theta Functions, by A. B. Coble. 1929.

Volume 11. The Theory of Approximation, by D. Jackson. 1930

Volume 12. Topology, by S. Lefschetz. 1930.

Volume 13. Foundations of Point Set Theory, by R. L. Moore. 1932.

Volume 14. Differential Equations from the Algebraic Standpoint, by J. F. Ritt. 1932.

Volume 15. Linear Transformations in Hilbert Space and their Applications to Analysis, by M. H. Stone. 1932.

Volume 16. Algebraic Functions, by G. A. Bliss. 1933.

Volume 17. Lectures on Matrices, by J. H. M. Wedderburn. 1934.

Volume 18. The Calculus of Variations in the Large, by M. Morse. 1934.

Volume 19. Fourier Transforms in the Complex Domain, by R. E. A. C. Paley and N. Wiener. In press. 